\title{
Effect of students' investigative experiments on students' recognition of interference and diffraction patterns: An eye-tracking study
}

\author{
Ana Susac $\odot,{ }^{1, *}$ Maja Planinic $\odot,{ }^{2}$ Andreja Bubic, ${ }^{3}$ Katarina Jelicic $\odot,{ }^{2}$ \\ Lana Ivanjek, ${ }^{4}$ Karolina Matejak Cvenic $\odot,{ }^{2}$ and Marijan Palmovic $\odot^{5}$ \\ ${ }^{1}$ Department of Applied Physics, Faculty of Electrical Engineering and Computing, \\ University of Zagreb, Unska 3, 10000 Zagreb, Croatia \\ ${ }^{2}$ Department of Physics, Faculty of Science, University of Zagreb, Bijenicka 32, 10000 Zagreb, Croatia \\ ${ }^{3}$ Chair for Psychology, Faculty of Humanities and Social Sciences, \\ University of Split, Sinjska 2, 21000 Split, Croatia \\ ${ }^{4}$ Physics Education Research, Faculty of Physics, Technische Universität Dresden, \\ Haeckelstraße 3, 01069 Dresden, Germany \\ ${ }^{5}$ Laboratory for Psycholinguistic Research, Department of Speech and Language Pathology, \\ University of Zagreb, Borongajska cesta 83h, 10000 Zagreb, Croatia
}

(Received 24 July 2020; accepted 24 December 2020; published 25 February 2021)

\begin{abstract}
Recognition of interference and diffraction patterns is a difficult task for both high-school and university students. Many students fail to observe important features of particular patterns and identify the differences among similar patterns. In this study, we investigated if performing students' investigative experiments can help high-school students in recognition of typical interference and diffraction patterns. Students in the experimental group were exposed to a teaching intervention that included five students' investigative hands-on experiments on wave optics whereas the control group had the standard lecture-based physics teaching. We measured eye movements of students from both the experimental and control groups while they were identifying patterns produced by monochromatic light on a double slit, single slit, and diffraction grating, and by white light on a diffraction grating. Students from the experimental group had a higher percentage of correct answers than students in the control group that indicated that students' investigative experiments had a positive effect on their recognition of interference and diffraction patterns. However, the low percentage of correct answers, even in the experimental group, confirms that distinguishing of the typical interference and diffraction patterns remains a difficult task for high-school students even if they had performed investigative hands-on experiments. Eye-tracking data showed that students from the experimental group had a shorter dwell on multiple-choice patterns, possibly because they were more familiar with interference and diffraction patterns and felt more confident in choosing the correct pattern. All students attended more to those patterns which they chose as the correct answer and that corroborates the previous findings. Overall, the results indicate that students' recognition of interference and diffraction patterns can be improved by introducing hands-on investigative experiments in the classroom.
\end{abstract}

DOI: 10.1103/PhysRevPhysEducRes.17.010110

\section{INTRODUCTION}

The recognition of typical interference and diffraction patterns is one of the expected learning outcomes of studying wave optics, as stated in standard textbooks (e.g., Ref. [1]). Moreover, students are expected to be able to sketch by themselves the typical interference and

\footnotetext{
*Corresponding author. ana.susac@fer.hr

Published by the American Physical Society under the terms of the Creative Commons Attribution 4.0 International license. Further distribution of this work must maintain attribution to the author(s) and the published article's title, journal citation, and DOI.
}

diffraction patterns [1]. The recognition of various interference and diffraction patterns is also included in several learning goals used in developing the item bank for measuring understanding of wave optics [2].

Although the recognition of typical interference and diffraction patterns may seem like a simple task, our teaching experience suggests that is a rather demanding task for students. The typical interference and diffraction patterns produced by monochromatic light on a double slit, single slit, and diffraction grating look very much alike to students who encounter them for the first time. The process underlying their recognition is not as quick and automatic as it is for that of socially important visual stimuli, such as faces (e.g., Refs. [3,4]). To be able to differentiate typical interference and diffraction patterns, students need to 
recognize the important features of particular patterns. The interference of light from two coherent sources (e.g., two narrow slits) generates a pattern of alternating maxima (bright fringes) and minima (dark fringes) on the screen. If the slits are very narrow and the screen is very distant, the fringes are equidistant and have a comparable intensity. The pattern on the screen produced by passing of monochromatic light through a diffraction grating also consists of equidistant maxima of similar intensity, but the maxima are now very narrow and intense whereas the dark regions between them are relatively wide. On the other hand, diffraction of monochromatic light on a single slit generates a pattern with a pronounced central maximum which is wider, and has a higher intensity, than the other maxima.

Students learn about the reasons why wave optics patterns look the way they do, and they also see them in textbooks and classroom demonstrations. However, when they are presented with several familiar patterns and need to choose the correct one, the task becomes more complex than simple recall and recognition. Unless students really understood the key features of each pattern, they will have trouble recognizing the correct pattern. The main benefit of remembering the pattern is that it represents an essential part of each phenomenon of wave optics. Unless students know the pattern and its key features it is hard to say that they know much about the phenomenon in question (e.g., it is questionable what meaning they can assign to the learned equations describing the phenomena if they do not know how the equations are related to the patterns to which they apply). The double-slit pattern is also important to know and understand when later studying the double-slit experiment with electrons in quantum mechanics.

The results of our previous eye-tracking study confirmed that the recognition of typical interference and diffraction patterns is a difficult task for students [5]. Only $20 \%$ of high-school students were able to recognize the double-slit interference pattern and the diffraction grating pattern of monochromatic light. They more often identified the single-slit diffraction pattern correctly, probably due to its distinguishable central maximum. The easiest task for students was recognizing the diffraction pattern of white light on an optical grating. Eye-tracking data suggested that even students who incorrectly answered this question mostly attended the colored patterns, thus indicating that they were aware that the diffraction grating separates white light into colors. Furthermore, eye tracking revealed that students who identified patterns correctly attended more the correct pattern than other options.

Many students who participated in our previous study could see these patterns only in their textbooks or in demonstration experiments performed by their teachers. Several factors that may prevent students from learning from teacher demonstrations were identified in a study of Roth et al., including "interference from other demonstrations and images that had some surface resemblance" and "lack of opportunities for students to test their descriptions and explanations" [6]. It has been suggested elsewhere that students should have a more active role in performing both demonstration and laboratory experiments [7].

In the present study, we decided to explore the effect of students' investigative experiments on their recognition of interference and diffraction patterns. To exclude possible confounding factors, we tested students who performed investigative experiments (the experimental group), and their peers from the same school who received the standard lecture-based teaching, including several demonstration experiments (the control group). Students in the experimental group performed experiments with laser light on a very narrow double slit, single slit, and diffraction grating followed by the experiment with the diffraction of white light on a diffraction grating.

To our knowledge, the present study is the first physics education research (PER) study using eye tracking to directly compare two teaching interventions, i.e., to compare visual attention of students in the experimental group who performed the investigative hands-on experiments and students in the control group who were taught in a standard lecture-based way.

In this study, we aimed to answer the following research questions:

(i) Do students from the experimental group better recognize and distinguish typical interference and diffraction patterns obtained by the double slit, single slit, and diffraction grating than their peers in the control group?

(ii) What is the difference in the visual attention between students in the experimental and control group on the level of questions and distractors?

Our hypothesis was that the investigative hands-on experiments would help students in recognizing the important features of the interference and diffraction patterns. We expected that students in the experimental group would be more efficient (more accurate and faster) in recognizing typical wave optics patterns.

\section{BACKGROUND}

Physics education research (PER) studies have shown that wave optics is a difficult topic for students. Previous studies conducted on university students $[2,8-16]$ revealed numerous student conceptual difficulties with interference and diffraction of light (for a detailed list see Ref. [2]). Similar difficulties were found in rather scarce PER studies on wave optics with high-school students $[5,17,18]$ or firstyear university students who did not take university courses covering wave optics $[19,20]$. One of the roles of highschool physics is preparing students for university physics [21], so high-school student understanding of wave optics needs to be further explored.

Some previous studies reported developing and implementing tutorials [11] and peer discussions [22], as well as 
using different visualizations [23] and representations [18] for teaching wave optics. Although different aspects of wave optics teaching and learning were investigated, student recognition of typical interference and diffraction patterns is not yet thoroughly studied. A previous study on measuring students' conceptual understanding of wave optics used two test items to probe university students' recognition of interference and diffraction patterns [2]. One of the questions was the most difficult one in the test with only about $10 \%$ of correct answers and the second question was also above average difficulty with about $35 \%$ of correct answers (it was in multiple-choice format with four distractors). These results indicate that identification of interference and diffraction patterns represents a demanding task for university students. As mentioned above, in our previous eye-tracking study we obtained similar results for high-school students who had the most difficulty in recognizing double-slit interference pattern and diffraction grating pattern of monochromatic light [5]. In this study, we used the same patterns as in the previous study [5], but we also added patterns produced by passing red laser light through a single slit, double slit, and diffraction grating. Furthermore, we compared the effect of two teaching interventions on the recognition of typical wave optics patterns.

The observation of a physical phenomenon is usually the first step in developing students' understanding of the phenomenon. Many inquiry-based interactive teaching methods, such as investigative science learning environment (ISLE) and the 5E (engage, explore, explain, elaborate, and evaluate) model, take this into account and propose observation of phenomena as an initial activity in a learning sequence [24,25]. Interference and diffraction of light are behind various phenomena that can be observed in everyday life, such as the colors on a soap bubble or the surface of a DVD. However, it is much easier to introduce and explore interference and diffraction through experiments with monochromatic light on a double slit, single slit, and diffraction grating followed by the diffraction of white light on a diffraction grating. The typical interference and diffraction patterns produced by monochromatic light on a double slit, single slit, and diffraction grating, and by white light on a diffraction grating can be found in all standard high-school and university textbooks [1,26-29].

Textbooks sometimes contain photographs from the experiments but important features of typical interference and diffraction patterns are often illustrated by graphs showing the intensity of typical interference and diffraction patterns [1,26-29]. Previous PER studies have identified student difficulties with graphs (e.g., Refs. [30,31]) and the use of multiple representations [32]. Kohl and Finkelstein found significantly different student performance on isomorphic quiz and homework problems that indicated their difficulties in switching between different representations, including graphical and pictorial representations of interference and diffraction patterns [32]. These results suggest that it is probably not enough just to see intensity plots and typical interference and diffraction patterns in the textbook to understand all the details and distinguish different patterns. The same authors found the effects of the instructional environment on students' representational skills [33].

In addition to using standard behavioral responses to multiple-choice questions on interference and diffraction patterns, we used eye tracking to obtain information on students' visual attention during pattern recognition. Eye tracking is increasingly used by PER researchers to investigate students' problem solving [34-50]. Results of several studies have shown differences in visual attention of students who correctly and incorrectly answered multiplechoice questions $[35,43,46]$. Students generally focus more on chosen answers than other options $[35,38,49]$ and from the time they spent inspecting particular options it is possible to infer which options are most attractive to them [5]. It was found that students who answered the question correctly spent more time attending relevant factors than their peers who answered incorrectly [36,43]. Further, several studies have shown that visual cues in a relevant part of the problem can direct students' attention and influence their reasoning, thus providing scaffolding in problem solving $[39,44,45]$. Previous PER studies also used eye tracking to compare the visual attention of different groups of students, e.g., physics and nonphysics students [36,37]. Han et al. used eye tracking to evaluate eye movements and students' performance in the pretest and the post-test condition [49]. Similarly, Brückner et al. [42] used pretest-post-test design in an eye-tracking study to evaluate the effect of attending first semester courses in physics and economics on student understanding of graphs. However, they could not directly compare the results of teaching interventions because the student populations were quite different in the two groups. In this study, we compared the effect of two different teaching interventions on students' recognition of wave optics patterns using two comparable groups of students.

\section{METHODS}

\section{A. Participants}

Participants in this study were 52 high-school students (age 18 years) in the last (fourth) year of high school in Zagreb, Croatia. All participants attended a general education type of high school (gymnasium) that typically prepares students for continuation of education at universities and where physics is taught as a compulsory subject throughout all four years of high school. Students had two 45-min physics lessons per week.

\section{B. Intervention}

The experimental group included 26 students (17 female, 9 male) who were subjected to a teaching intervention whose purpose was to pilot the developed 
teaching sequence and materials that will later be applied on a larger sample of students in our research project. The teaching intervention included five students' investigative hands-on experiments. The covered topics in eight 45-min periods were interference, diffraction, and polarization of light. Of the five investigative experiments, four were related to interference and diffraction of light (one was related to polarization). These experiments included students' investigation of interference pattern obtained with laser light and double slit, investigation of optical grating pattern with monochromatic and white light, and investigation of diffraction pattern obtained with laser light on a single slit. The first experiment included data collection, whereas the other three were qualitative investigations (due to the limited available time). Students worked in groups of 4-5 and were provided with equipment and investigation questions for each experiment. They were required to set up the experiments and to answer the investigation questions. The results were discussed with the whole class. Each experiment took 25-45 min of class time. In addition to students' hands-on experiments, students were also shown several demonstrations of the double slit, single slit, and diffraction grating patterns which were used as observational experiments when new phenomena were introduced. Such demonstration experiments are not uncommon in gymnasium physics teaching in Croatia; however, students' hands-on investigative experiments are much less common. Also, the quality of the school demonstration equipment is often poor and does not allow for a clear distinction between patterns when passing laser light through a double slit and a single slit. The teaching intervention was performed by a member of the research group from the Faculty of Science (K. J.), who is also an experienced high-school physics teacher.

The control group included 26 students (13 female, 13 male), from a different class of the same school, that did not participate in the intervention. Both the control and experimental group were taught by the same regular physics teacher before the intervention. The control class had similar average grade in physics $(3.6 \pm 1.1)$ as the experimental class $(3.5 \pm 1.1)$. The control group received the standard lecture-based physics teaching on wave optics including experiments performed by their regular teacher (demonstration experiment with two lasers of different wavelength and two diffraction gratings with different grating constant, and a demonstration experiment showing diffraction of white light and monochromatic light on a single slit). Besides demonstration experiments performed by the teacher, students were shown on presentation slides the patterns obtained by interference and diffraction of green laser light on a double and single slit followed by patterns obtained by passing red laser light and white light through a diffraction grating.

\section{Materials}

Seven multiple-choice questions on interference and diffraction patterns were used in this study. The questions were the following:

Q1: Which of the patterns on the screen was produced by the interference of red laser light from two coherent sources? [Possible answers were four red laser light patterns shown in Fig. 1(a)]

Q2: Which of the patterns on the screen was produced by passing red laser light through a single slit? [Possible answers were four red laser light patterns shown in Fig. 1(a)]

Q3: Which of the patterns on the screen was produced by passing red laser light through a diffraction grating? (Possible answers were four red laser light patterns shown in Fig. 1(a)]

Q4: Which of the patterns on the screen was produced by passing white light through a diffraction grating? [Possible answers were two grey and two rainbow patterns shown in Fig. 1(b)]

Q5: Which of the patterns on the screen was produced by the interference of green light from two coherent sources? [Possible answers were four green light patterns shown in Fig. 1(c)]

(a)
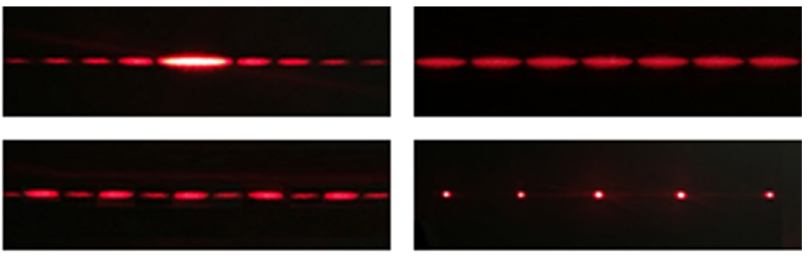

(b)
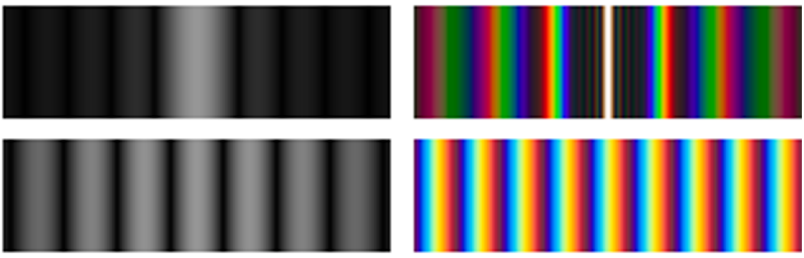

(c)
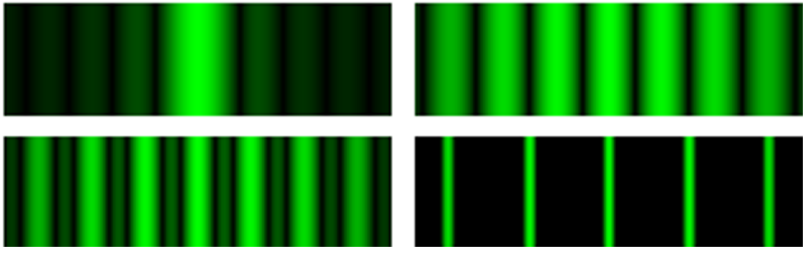

FIG. 1. (a) Red laser light patterns obtained by the single slit, double slit, three slits, and diffraction grating were possible answers for question Q1-Q3. (b) Four patterns were possible answers for question Q4 where students were asked to recognize pattern produced by passing white light through a diffraction grating (gray single-slit pattern, correct pattern, gray double-slit pattern, rainbow fringes). (c) Green light patterns obtained by the single slit, double slit, three slits, and diffraction grating were possible answers for question Q5-Q7. 
Q6: Which of the patterns on the screen was produced by passing green light through a single slit? [Possible answers were four green light patterns shown in Fig. 1(c)]

Q7: Which of the patterns on the screen was produced by passing green light through a diffraction grating? [Possible answers were four green light patterns shown in Fig. 1(c)]

The red laser light patterns [Fig. 1(a)] were photographed from the experiments and modified, and they corresponded to patterns that students in the experimental group saw during the intervention (except the third pattern which represents pattern obtained by three slits). The white light and green light patterns were taken from the website of Dietrich Zawischa [51] and modified [Figs. 1(b) and 1(c)]. The first three questions $(\mathrm{Q} 1-\mathrm{Q} 3)$ were presented to participants in counterbalanced order, followed by question Q4, and finally, the last three questions (Q5-Q7) were presented in counterbalanced order. The order of the appearance of four patterns in questions Q1-Q3 and Q5-Q7 was also counterbalanced.

\section{Procedure}

Eye-movement data were recorded using the SMI screen-based RED-m system (SensoMotoric Instruments G.m.b.H.) with sample rate $120 \mathrm{~Hz}$ integrated with 17 " TFT LCD monitor. The participants were free to move their head during the measurements within the region in space where their eyes were inside the eye tracker's field of view. The eye-tracking system was calibrated for each participant before the data recording using a 5-point calibration algorithm. Questions were presented on a monitor at the distance of $50 \mathrm{~cm}$ from the participants' eyes. The size of each pattern on the screen was $10 \mathrm{~cm} \times 3 \mathrm{~cm}$. By choosing the answer, participants advanced to the next question. There was no time limit to answer the questions.

After the measurement of eye movements, students were asked to sketch and describe the patterns on the screen produced by the following:

(a) the interference of light from two coherent sources,

(b) passing monochromatic light through a single slit,

(c) passing monochromatic light through a diffraction grating,

(d) passing white light through a diffraction grating.

Figure 2 shows an example of one student's sketches and descriptions. The whole procedure, including preparation, eye-movement calibration and recording, and answering the paper-and-pencil questions about typical interference and diffraction patterns lasted around $20 \mathrm{~min}$.

\section{E. Data analysis}

Students' responses to the seven multiple-choice questions described above were scored correct or incorrect. In addition, these scores were corrected independently by two of the authors, taking into consideration students' sketches and descriptions. If a correct answer during the eyetracking measurement was given with a correct sketch and/or description in the paper-and-pencil test, the student was awarded 1 point. If a correct answer was given with a wrong or no sketch and description, the student was

(a) prolaskom monokromatske svjetlosti kroz optičku rešetku

Skica: Opis:

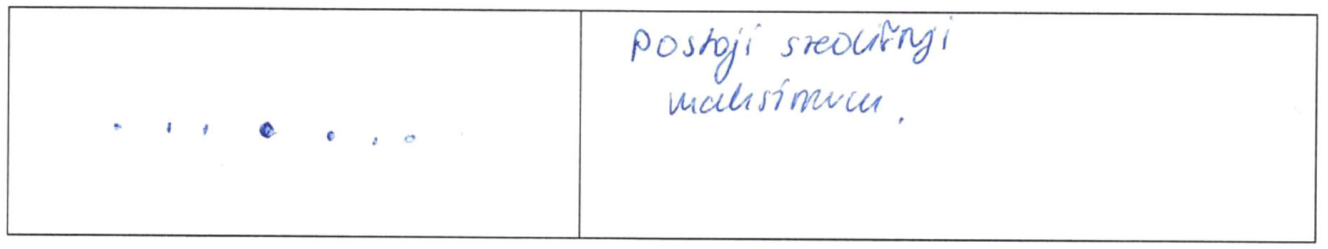

(b) prolaskom bijele svjetlosti kroz optičku rešetku

Skica: Opis:

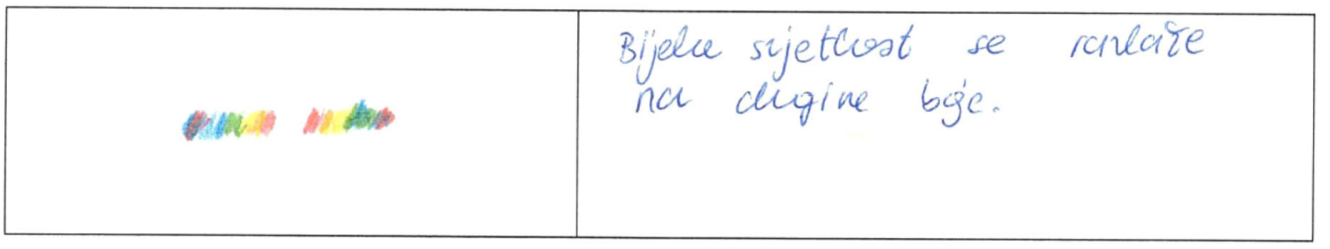

FIG. 2. An example of one student's sketch and description for the pattern produced by passing monochromatic light through a diffraction grating ("there is a central maximum") and by passing white light through a diffraction grating ("white light decomposes into rainbow colors"). 
awarded 0 points. A correct answer without a correct sketch and/or description indicated that the correct answer was probably selected by chance or for a wrong reason. The agreement between the raters was very high (98\%) and the differences in scoring have been discussed and consensually resolved. Students' scores after the correction are reported in this paper.

The recorded eye movements data were analyzed using BeGaze software that allows evaluation of the eye fixations and saccades. Fixation is the state when the eye is stationary over a period of time, while saccade is the rapid eye movement between fixations. BeGaze used the identification by dispersion-threshold (IDT) algorithm to determine fixations with maximum dispersion value $100 \mathrm{px}$ and minimum fixation duration $80 \mathrm{~ms}$.

Calibration data and visual inspection of scan paths showed a reasonably good quality of eye-tracking data for most participants. The data from one participant were not recorded for all seven questions and the data from another two participants indicated inadequate calibration. As such, the eye-tracking data from these three participants (two from the experimental group and one from the control group) were not included in the further analysis.

We defined five rectangular areas of interest (AOIs) for each question that included the text of the problem (AOI question) and multiple-choice answers (a, b, c, and d). We also used AOI patterns which covered all four multiplechoice answers.

As our previous studies showed, average fixation duration is rather constant for a specific representation (such as graphs, wave optics patterns) and does not show much variation for different corresponding tasks $[5,40]$. As a result, dwell time and number of fixations are highly correlated and show a similar pattern of responses. Consequently, although we analyzed several eye tracking variables, i.e., dwell time (viewing time), number of fixations, average fixation duration, and number of revisits (returns to previously inspected AOI), we report only a portion of results of comparison of their values for the students in the experimental and control group.

To compare the results of students in the experimental and control group, Student's $t$ tests and $\chi^{2}$ tests were conducted. Several one-way and two-way analyses of variance (ANOVAs) and Tukey's honestly significant difference (HSD) tests were conducted. A threshold of $p=0.05$ was used for determining the level of effect significance within all conducted tests.

\section{RESULTS}

\section{A. Analysis of students' scores}

The percentage of correct answers of students (after correction) in the experimental group $(31 \pm 28) \%$ was higher than the percentage of correct answers of students in the control group $(17 \pm 21) \%[t(50)=2.08 ; p<0.05]$. Percentages of correct answers of students in the experimental and control group on all questions are shown in Fig. 3. The $\chi^{2}$ tests showed a statistically significant difference in frequencies of correct answers between the two groups only for the question regarding the pattern produced by passing white light through a diffraction grating $\left[\chi^{2}(1)=13.50, p<0.001\right]$. Students from the experimental group had higher scores on that question than students in the control group.

The distribution of students' responses to each question is shown in Table I. The percentage of correct answers was highest for the question regarding the pattern produced by passing white light through a diffraction grating, on which $79 \%$ of students chose the correct pattern. After the correction based on students' paper and pencil sketches and descriptions, we can conclude that $60 \%$ of students were able to identify the diffraction pattern of white light on a diffraction grating. Furthermore, around $20 \%$ of students (after correction) could recognize the single-slit patterns and the diffraction patterns of monochromatic light on a diffraction grating. The results indicate that students had

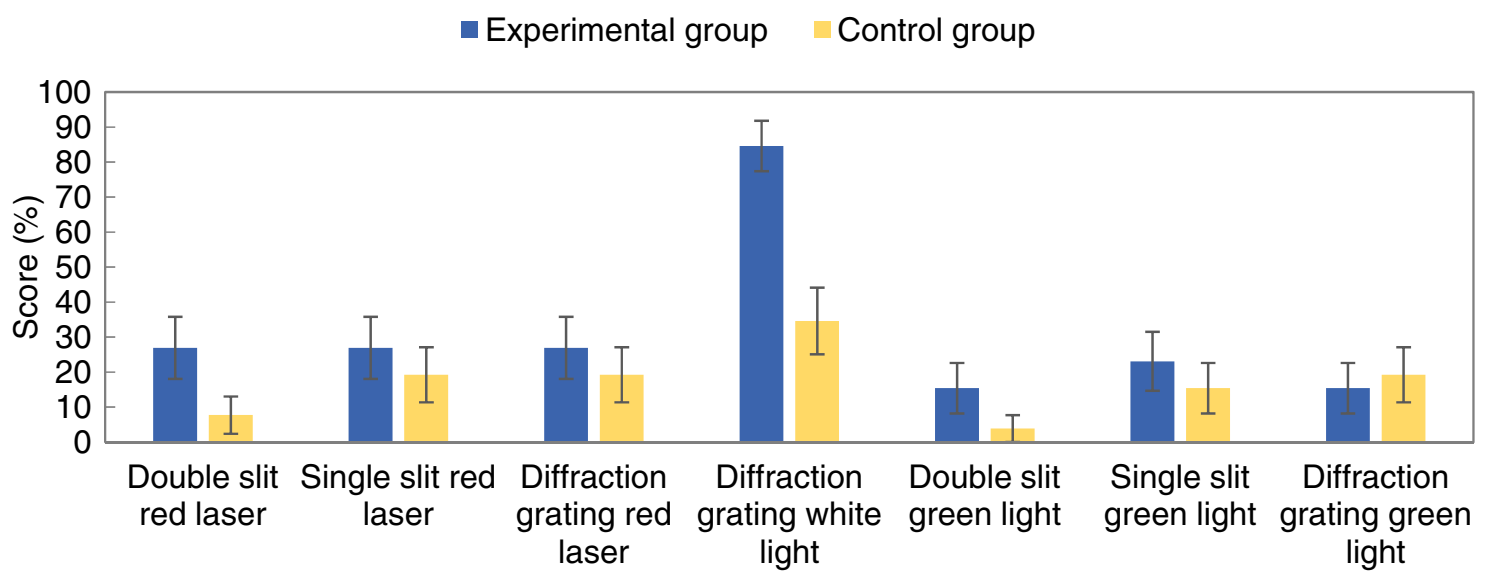

FIG. 3. Scores of students in the experimental and control group on all seven questions. The error bars represent 1 standard error of the mean (SEM). 
TABLE I. The percentages of students that selected a particular multiple choice (single-slit pattern, double-slit pattern, three-slits pattern, diffraction grating pattern) in each question. The correct answer is in bold. The values in parentheses are the percentages of the correct answers after the correction based on students' paper and pencil answers.

\begin{tabular}{lcccc}
\hline \hline & \multicolumn{3}{c}{ Multiple choice $(\%)$} \\
\cline { 2 - 5 } Question & Single slit & Double slit & Three slits & Diffraction grating \\
\hline Double slit & 27 & $\mathbf{2 5}(\mathbf{1 7})$ & 27 & 21 \\
Single slit & $\mathbf{4 0}(\mathbf{2 3})$ & 29 & 6 & 25 \\
Q3 Diffraction grating red laser & 27 & 25 & 6 & $\mathbf{4 2}(\mathbf{2 3})$ \\
Double slit & 23 & $\mathbf{2 1}(\mathbf{1 0})$ & 2 & 15 \\
Single slit & $\mathbf{5 4}(\mathbf{1 9})$ & 12 & 8 & $\mathbf{3 3}$ \\
Q7 Diffraction grating green light & 44 & Diffraction grating & Gray double slit & Rainbow stripes \\
Q4 Diffraction grating white light & Gray single slit & $\mathbf{7 9}(\mathbf{6 0})$ & 8 & 10 \\
\hline \hline
\end{tabular}

the most difficulty identifying double-slit interference patterns. Table II suggests that students were most likely to choose the single-slit pattern as an answer in four out of six questions (Q1, Q2, Q6, and Q7).

Students in the experimental group performed experiments with red laser, so we compared students' scores on questions regarding red laser patterns and green light patterns for the experimental and control group (Fig. 4). We conducted a two-way mixed-design ANOVA with factors the type of light (red laser vs green light) and group (experimental vs control). There were no significant main effects of the type of light $[F(1,50)=2.89, p>$ $\left.0.05, \eta_{p}^{2}=0.055\right]$ and group $[F(1,50)=1.32, p>0.05$, $\left.\eta_{p}^{2}=0.026\right]$. The interaction effect $[F(1,50)=0.89$, $\left.p>0.05, \eta_{p}^{2}=0.018\right]$ was also not statistically significant. However, Fig. 4 shows a trend that students in the experimental group had higher scores on questions regarding red laser patterns than on questions regarding green light patterns.

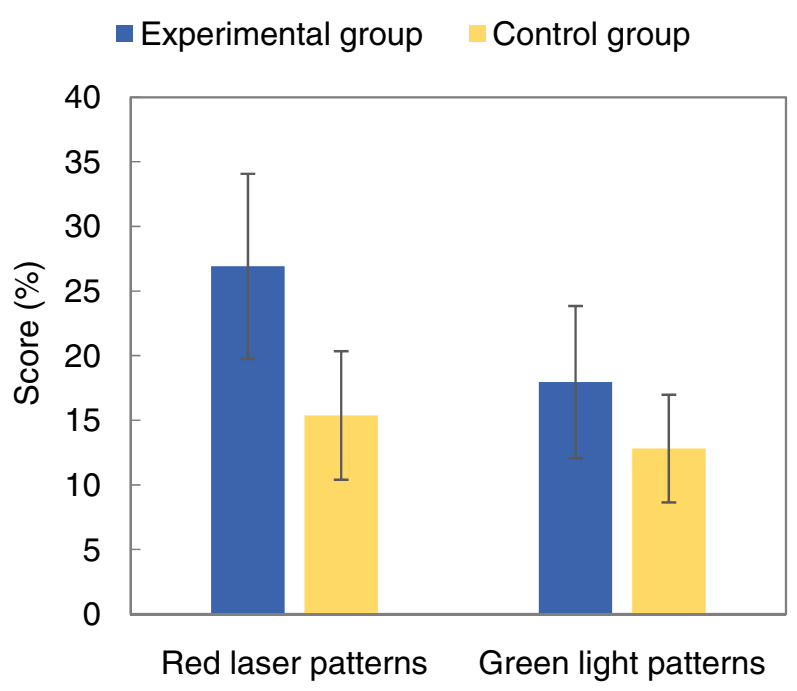

FIG. 4. Mean scores of students in the experimental and control group on questions regarding red laser patterns and green light patterns. The error bars represent 1 standard error of the mean (SEM).

\section{B. Analysis of eye-tracking data}

Figure 5 shows the total dwell time of students in the experimental and control group on all questions. To compare the results for the experimental and control group, we conducted a two-way mixed-design ANOVA with factors of question (Q1-Q7) and group (experimental vs control). There were no significant main effects of question $\left[F(6,282)=1.43, \quad p>0.05, \quad \eta_{p}^{2}=0.029\right]$ and group $\left[F(1,47)=1.41, p>0.05, \eta_{p}^{2}=0.029\right]$. The interaction effect $\left[F(6,282)=0.67, p>0.05, \eta_{p}^{2}=0.014\right]$ was also not statistically significant.

To further explore visual attention of students in the two groups, we evaluated dwell times for AOI question and AOI patterns (Fig. 6). To compare the dwell times for the experimental and control group, we conducted a two-way mixed-design ANOVA with factors AOI (question vs patterns) and group (experimental vs control). The results showed a statistically significant main effect of both factors, AOI $\left[F(1,6)=111.81, p<0.001, \eta_{p}^{2}=0.949\right]$ and group $\left[F(1,6)=7.11, p<0.05, \eta_{p}^{2}=0.542\right]$, and their interaction was marginally statistically significant $\left[F(1,6)=5.93, p=0.05, \eta_{p}^{2}=0.497\right]$. Students attended more AOI patterns than AOI question, and students from the control group attended more AOI patterns than their peers from the experimental group.

The next step in the eye-tracking analysis was a comparison of the distribution of the visual attention of students in the experimental and control group across multiple-choice patterns. For only three questions was the distribution statistically different for the experimental and control group (Fig. 7), and here we report the corresponding results. We conducted two-way mixeddesign ANOVAs with factors group (experimental vs control) and pattern (e.g., single slit vs double slit vs three slits vs diffraction grating). The results are shown in Table II. Students from the experimental group had a smaller number of fixations and revisits than students from the control group (the difference of fixation numbers for the question Q1 was not statistically significant at the level $p<0.05)$. 


\section{Experimental group $\square$ Control group}

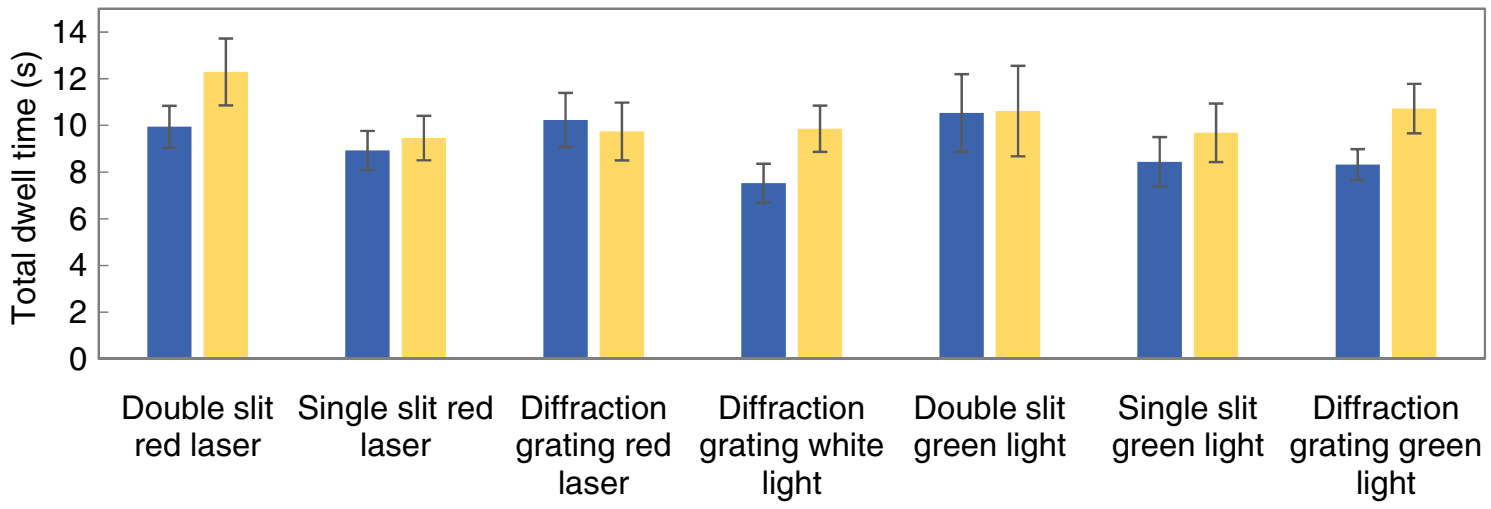

FIG. 5. The total dwell time of students in the experimental and control group on all seven questions. The error bars represent 1 standard error of the mean (SEM).

Students had different numbers of fixations and revisits for different patterns. For the interference of red light on the double slit (question Q1), students had a larger number of fixations on the single-slit pattern than diffraction-grating pattern $(p<0.05)$. The corresponding number of revisits was larger for the single-slit pattern than the three-slits pattern and diffraction-grating pattern $(p<0.05$ and $p<0.01$, respectively), and it was also larger for the double-slit pattern than the three-slits pattern and diffraction grating pattern (both $p<0.01$ ). For the question Q4, students had the largest number of fixations and revisits on the correct pattern (all pairwise comparisons were statistically significant at the level $p<0.01$ ), and additionally, the number of revisits was larger on the gray single-slit pattern than the gray double-slit pattern. For the question Q7, students had a larger number of fixations on the diffraction grating pattern than the three-slits pattern $(p<0.01)$. The corresponding number of revisits was the

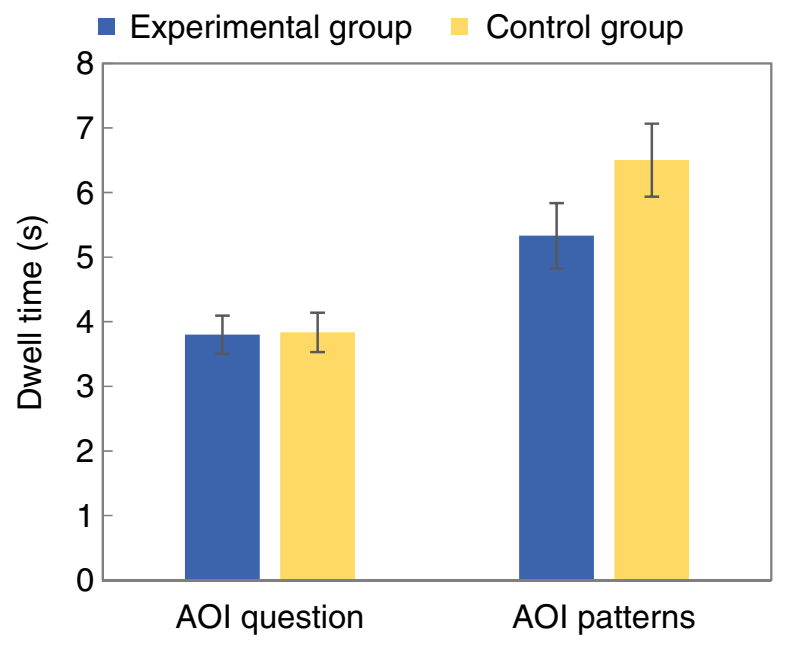

FIG. 6. Mean dwell times of students in the experimental and control group for AOI question and AOI patterns. The error bars represent 1 SEM. largest for the diffraction grating pattern $(p<0.01$ for comparisons with the single-slit and the three-slits patterns, and $p<0.05$ for the comparison with the double-slit pattern).

Finally, we investigated the relationship between students' responses and their eye movements. Figure 7 suggests that students most attend to the patterns which they identify as correct. On the question regarding the pattern produced by passing of white light through a diffraction grating, students attended most to the correct pattern which they also most often chose as their answer (Table I). Similarly, on the question regarding the pattern produced by passing green light through a diffraction grating, students spent most time attending the correct pattern and the single-slit pattern, which were also preferred options (Table I). We further examined this notion by splitting the students into groups, according to their responses, and comparing their dwell times. The results confirmed that students attended most to the patterns which they identified as correct. Since this was not the main aim of our study, we reported the results for one question in Appendix A for illustration of this conclusion.

\section{DISCUSSION}

The analysis of students' scores has shown that students from the experimental group had a higher percentage of correct answers than students in the control group, thus indicating that students' investigative experiments had a positive effect on their recognition of interference and diffraction patterns. The low percentage of correct answers, even in the experimental group, confirms that distinguishing the typical patterns obtained by interference and diffraction of light on a double slit, single slit, and diffraction grating is a difficult task for high school students. This finding corroborates our previous results [5] and the results from the PER studies on university students which used a couple of multiple-choice questions with interference and diffraction patterns $[8,28]$. 
(a) Q1 Double slit red light
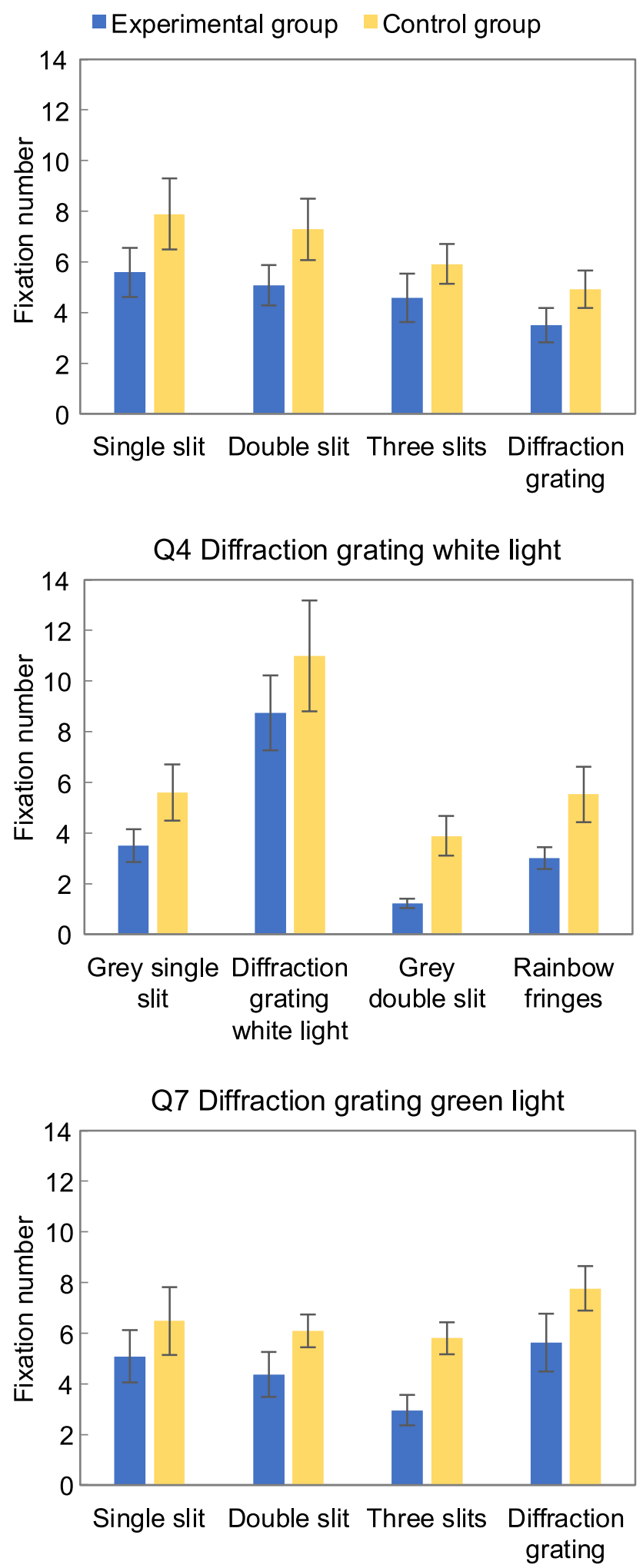

(b)

Q1 Double slit red light
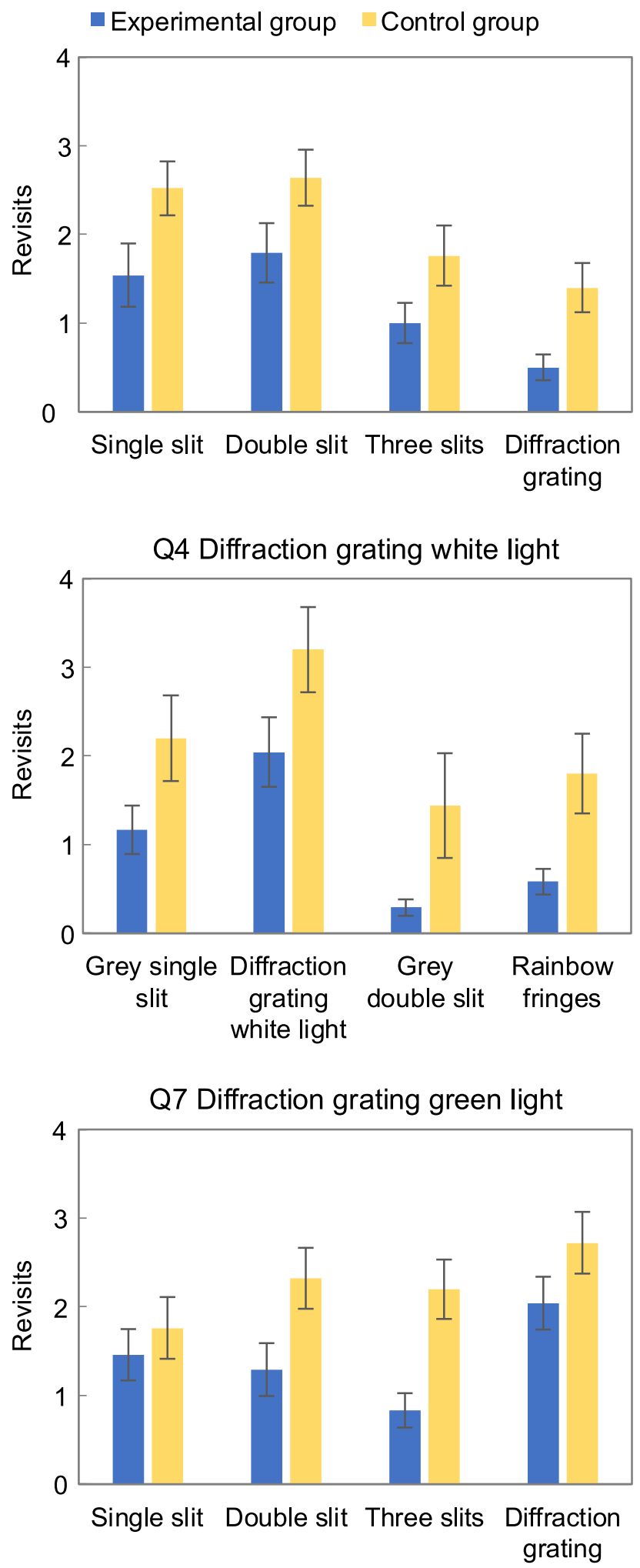

FIG. 7. (a) Distribution of the fixation numbers of students in the experimental and control group across multiple-choice patterns for three questions: double slit, Q4 (diffraction grating white light), and Q7 (diffraction grating green light). (b) Distribution of the number of revisits of students in the experimental and control group across multiple-choice patterns for the same questions. The error bars represent 1 SEM. 
TABLE II. Results of a two-way mixed-design ANOVAs with factors group (experimental vs control) and pattern (e.g., single slit vs double slit vs three slits vs diffraction grating), conducted for a number of fixations and revisits for questions Q1, Q4, and Q7.

\begin{tabular}{|c|c|c|c|c|c|c|c|c|c|}
\hline & \multicolumn{3}{|c|}{ Factor group } & \multicolumn{3}{|c|}{ Factor pattern } & \multicolumn{3}{|c|}{ Interaction } \\
\hline & $F(d f)$ & $p$ & $\eta_{p}^{2}$ & $F(d f)$ & $p$ & $\eta_{p}^{2}$ & $F(d f)$ & $p$ & $\eta_{p}^{2}$ \\
\hline Q1 fixations & $3.73(1,47)$ & 0.06 & 0.07 & $3.53(3,141)$ & 0.02 & 0.07 & $0.18(3,141)$ & $>0.05$ & 0.004 \\
\hline Q4 fixations & $5.61(1,47)$ & 0.02 & 0.11 & $29.70(3,141)$ & $<10^{-4}$ & 0.38 & $0.05(3,141)$ & $>0.05$ & 0.001 \\
\hline Q7 fixations & $6.13(1,47)$ & 0.02 & 0.12 & $4.61(3,141)$ & 0.004 & 0.09 & $0.48(3,141)$ & $>0.05$ & 0.01 \\
\hline Q1 revisits & $7.77(1,47)$ & 0.008 & 0.14 & $13.20(3,141)$ & $<10^{-4}$ & 0.20 & $0.08(3,141)$ & $>0.05$ & 0.002 \\
\hline Q4 revisits & $5.74(1,47)$ & 0.02 & 0.11 & $17.23(3,141)$ & $<10^{-4}$ & 0.27 & $0.04(3,141)$ & $>0.05$ & 0.001 \\
\hline Q7 revisits & $5.28(1,47)$ & 0.03 & 0.10 & $7.42(3,141)$ & $10^{-4}$ & 0.14 & $2.59(3,141)$ & 0.05 & 0.05 \\
\hline
\end{tabular}

Students in the experimental group had the highest score on the question regarding the pattern produced by passing of white light through a diffraction grating, and the difference between the two groups was most pronounced on that question. This result confirms the previous conclusion that the easiest task for students was recognizing the diffraction pattern of white light on an optical grating [5] because they knew that diffraction grating separates white light into colors. It is possible that students memorize and recall better colorful patterns because they are more visually attractive, suggesting the importance of top-down processing in guiding visual attention. Generally, such processing is influenced by expectations and prior knowledge. For example, if the question is to identify a pattern produced by the interference of light from two coherent sources, students' prior knowledge and experience with the pattern may guide their processing - they would look for particular features. However, it is also possible that bottomup processing has an important role in directing students' visual attention to colorful patterns, that is in some respect similar to visual cues which can direct students' visual attention during problem solving $[39,44,45]$. Such processing starts with the perception of incoming stimulus (in our case wave optics pattern) that initiates and determines the higher-level processes involved in their recognition. Paying attention to colorful patterns would then represent an example of bottom-up processing. In any case, the results indicate that performing a hands-on experiment significantly improves students' recognition of the diffraction pattern of white light on an optical grating. Consequently, instructors should be aware of both approaches because probably most students use a combination of top-down and bottom-up processing.

Furthermore, it seems that students most often chose the single-slit pattern (as both correct and incorrect answer), possibly because of its prominent central maximum (Table I). They also attended the single-slit pattern the most (Fig. 9 in Appendix B). Again, this result is in agreement with results from our previous study [5], where students more often correctly identified the single-slit diffraction pattern than interference or diffraction pattern of monochromatic light on a double slit and diffraction grating. The percentage of correct answers on the question regarding diffraction of green light on a single slit was higher in the previous study because it was not corrected based on students' paper and pencil answers. Nevertheless, the results are quite comparable if no correction is used. Similar to the colors in the diffraction pattern of white light on an optical grating, the broad central maximum in the single-slit diffraction pattern is a characteristic feature that attracts students' visual attention.

There were no statistically significant differences in frequencies of correct answers between the two groups in questions regarding monochromatic patterns, probably due to the floor effect (the scores of students from both groups were rather low on these questions).

Possibly for the same reason, there was no statistically significant difference between students' scores on questions regarding red and green laser light patterns. As students in the experimental group performed hands-on experiments with red laser light, we could expect they would obtain higher scores on questions regarding red laser patterns compared to their peers. Indeed, the results show the anticipated trend, but the differences did not reach statistical significance.

The analysis of students' eye-tracking data provided further insight into the differences between the experimental and control group. Although Fig. 5 suggests longer total dwell times of experimental than the control group for some questions, the difference was not statistically significant. However, the comparison of dwell times for AOI question and AOI patterns (Fig. 6) revealed that students from the experimental group had a shorter dwell time for AOI patterns than their peers from the control group, whereas there was no difference between two groups' dwell times for AOI question. Almost equal dwell times for AOI question indicate that there are no differences between the groups as such, whereas shorter dwell time of experimental group for AOI patterns suggests that students who conducted hands-on experiments possibly felt more familiar with interference and diffraction patterns and more confident in choosing the correct pattern. This result corresponds to the previous finding that more efficient participants "know where to look" [52]. In two previous 
eye-tracking studies, researchers assessed students' response confidence and they found that students with low confidence ratings attended longer relevant task areas than confident students $[38,41]$. In this study, we also have some indications that lower dwell times are correlated with higher confidence, but additional data collection (i.e., assessment of confidence ratings) is needed to confirm these findings.

Further analysis of the distribution of the fixations across multiple-choice patterns confirmed that students in the experimental group had a smaller number of fixations than students from the control group on questions regarding recognition of patterns produced by the passing of white and green light through a diffraction grating. The results reveal which multiple-choice options the students attended the most. For the question regarding white light passing through a diffraction grating, students evidently attended most to the correct pattern. For the question regarding green light passing through a diffraction grating, students tended to fixate most on the diffraction grating pattern (correct answer). The eye-tracking data are in line with behavioral responses; students attended more those options that they chose as the correct answer. This relationship between visual attention and students' responses was reported in previous studies $[5,35,38,43,46,49]$. This was further confirmed in this study by comparing dwell times for multiplechoice patterns according to students' answers. Again, the results corroborate that students attend most the patterns which they identify as correct.

Analysis of revisits showed that students in the experimental group more rarely returned to the already inspected pattern that again implied that they knew where to look. On the other hand, the larger number of revisits for students in the control group indicated that they moved their attention from one distractor to another more while trying to decide which one is correct. So, eye tracking gave an insight into differences in students' strategies during recognition of wave optics patterns.

Moreover, the mean dwell time and mean number of fixations per distractor was rather low (1.5 s and 5-6 fixations) and the number of revisits per distractor was around 1.5-2 indicating that students did not analyze individual patterns for a very long time. They grasped features of patterns in several fixations, similarly as two fixations suffice in face recognition [3]. The eye-tracking data suggest that the recognition process was quite fast for all students participating in this study. However, students in the experimental group were more accurate and faster in recognizing typical wave optics patterns. This implies that teaching intervention with investigative experiments helped students to become more proficient in recognizing wave optics patterns than their peers who received the standard lecture-based teaching.

Furthermore, in this study we investigated the differences in the visual attention of students in the experimental and control group on the level of questions and distractors. We have also looked for a possible difference in the distribution of visual attention on the level of single patterns but did not find any statistically significant differences. The majority of participants looked most of the time around the center of the patterns. Such a finding is not surprising because the size of the patterns was not very large and, looking in the center of the patterns, students could grasp all important features. As mentioned above, students did not attend to individual patterns for a very long time but were deciding between different distractors. All this probably contributed to the fact that no reliable difference between the two groups in the distribution of attention within the distractor could be found.

The results of the study suggest that the inclusion of students' investigative experiments showed some benefits for students' ability to recognize typical interference and diffraction patterns, although the differences between the experimental and control group are smaller than expected. One factor that may have influenced the size of that difference is the fact that the study was conducted two months after the intervention was over, so students may have already forgotten some of the details of the experiments. Another important factor was that the intervention that was conducted was only in its pilot phase, in which the teaching materials and the teaching sequence itself were investigated and were not yet in their final form-some shortcomings were noticed in that process, which resulted in many changes that will be implemented for later research on a larger sample. However, the difference between the experimental and control group found in this study is significant, despite these factors.

The study opens up many questions. The first is the question of what is needed to get students to truly observe the physics phenomena and learn to notice and describe the differences among similar patterns. It can be hypothesized that the monochromatic interference and diffraction patterns were perceived by the students as very similar and that they did not succeed in such a short time frame (8 lesson periods) to notice and remember the key differences. Similar problems were documented in a previous study concerning student understanding of spectra, in which was found that students have difficulty distinguishing the properties of discrete line spectra and diffraction pattern [53]. Also, all students did not participate with the same level of involvement in the hands-on experiments and their relatively large number in each workgroup may have prevented some from more active exploration. Another question that comes up is why students choose some patterns and how do they choose them at all? The striking difference between the number of students who recognized correctly the white light diffraction grating pattern (the only pattern that included several colors) and the number of students who recognized the monochromatic interference and diffraction patterns correctly may suggest a partial 
answer to this question: students seem to remember better the patterns that visually stand out, that are colorful and/or have some other prominent features. This was also suggested in the finding that the most often chosen wrong answer was the single-slit diffraction pattern which has a broad central maximum that maybe made a stronger impression on the students than the equidistant patterns. The last remaining question may be how important it is that students correctly recognize different patterns. We believe that systematic and detailed observation of new physics phenomena is an important step in developing physics knowledge. We have often noticed in our teaching practice that students (even at the university level, and even more at the high-school or elementary school level) need to be taught to systematically and more deeply observe phenomena-otherwise they often remain at the level of noticing only the most prominent features, but fail to notice other important features. Even though the difference between the experimental and control group scores in this study was not large, and in both cases students' ability to recognize the studied phenomena was at a rather low level, we believe that it does provide the incentive to use more investigative experiments in the classroom, as they may provide students with better knowledge about phenomena. We will continue to further refine and develop our teaching intervention, and implement it on a larger sample of students, which may provide more insight in its effectiveness.

\section{ACKNOWLEDGMENTS}

This work has been supported in part by the Croatian Science Foundation under the Project No. IP-201801-9085.
TABLE III. Results of the one-way ANOVAs conducted on dwell times on the multiple-choice patterns (single slit, double slit, three slits, and diffraction grating) for four groups of students according to their answers to the question to recognize the pattern produced by the interference of red laser light from two coherent sources.

\begin{tabular}{lccc}
\hline \hline & $F(d f)$ & $p$ & $\eta_{p}^{2}$ \\
\hline Answered: single slit & $7.04(3,39)$ & $<0.001$ & 0.351 \\
Answered: double slit & $5.82(3,33)$ & 0.003 & 0.346 \\
Answered: three slits & $12.48(3,36)$ & $<0.001$ & 0.510 \\
Answered: diffraction grating & $5.71(3,27)$ & 0.004 & 0.388 \\
\hline \hline
\end{tabular}

\section{APPENDIX A: STUDENTS ATTEND MOST THE PATTERNS WHICH THEY IDENTIFY AS CORRECT}

We report here the results of the comparison of students' dwell times on the multiple-choice patterns according to their response to the question. We report the results for the question in which students were asked to identify the pattern produced by the interference of red laser light from two coherent sources because for this question the distribution of students' responses was rather uniform across the patterns (Table I; 27\% chose the single-slit pattern, 25\% chose the correct double-slit pattern, $27 \%$ chose the threeslits pattern, and the remaining $21 \%$ chose the diffraction grating pattern).

Table III shows the results of four one-way ANOVAs conducted independently for each group of students (split into groups according to their responses). The results of pairwise comparisons are shown in Fig. 8. The results indicate that students most attend to the patterns which they

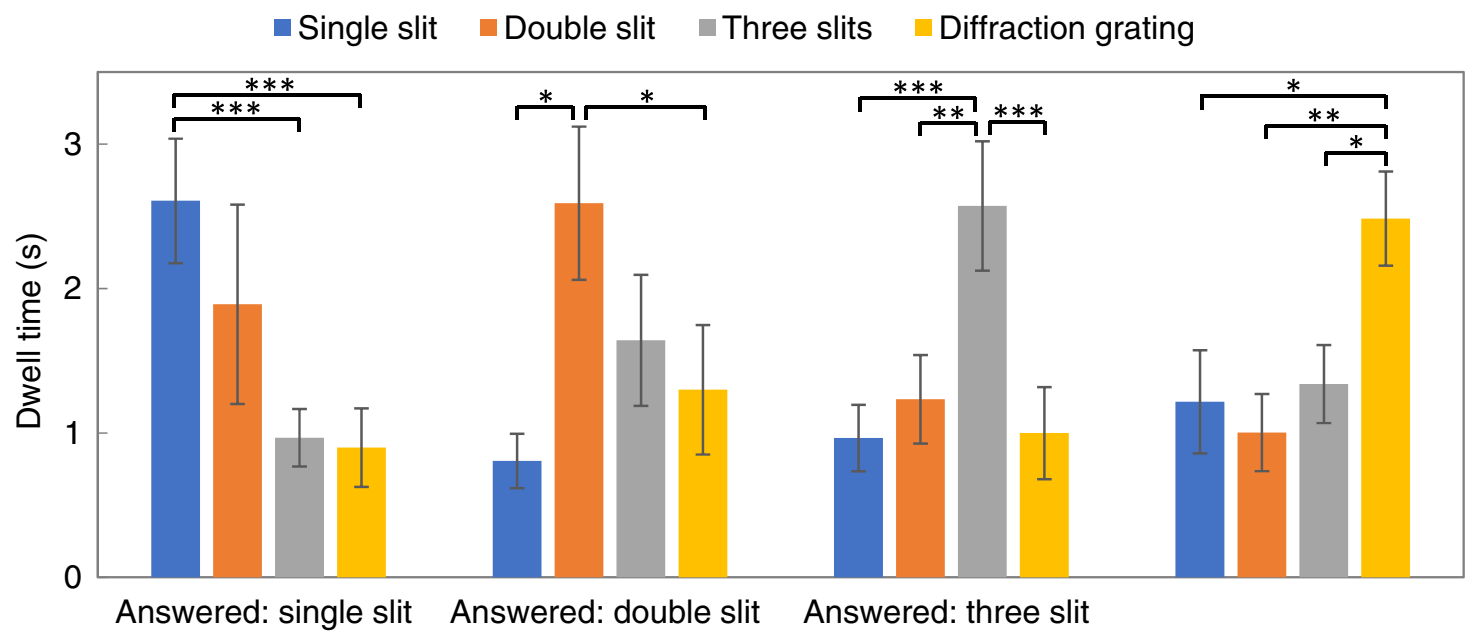

FIG. 8. Dwell times for multiple-choice patterns (single slit, double slit, three slits, and diffraction grating), evaluated separately for students according to their answers to the question to recognize the pattern produced by the interference of red laser light from two coherent sources. One or two asterisks indicate significantly different pairwise comparisons at levels of $p<0.05$ or $p<0.01$, respectively. 
identify as correct (all pairwise comparisons showed that the differences were statistically significant except dwell times on single-slit and double-slit patterns for students who answered single slit, and dwell times on double-slit and three-slits patterns for students who answered double slit.).

\section{APPENDIX B: Students attend the most to the single-slit pattern}

In three questions (Q5, Q6, Q7) students were asked to recognize patterns produced by the interference of green light from two coherent sources, single-slit pattern, and diffraction grating pattern among four green patterns shown in Fig. 1(c). Figure 9 shows the total dwell time, i.e., total time that students spent attending particular AOI while answering the three questions. It is evident that participants attended for the longest time to the single-slit diffraction pattern. The corresponding one-way ANOVA showed that pattern had a statistically significant effect on total dwell time $\left[F(3,144)=4.20, \quad p=0.007, \eta_{p}^{2}=0.080\right]$. The difference between total dwell time on the single-slit

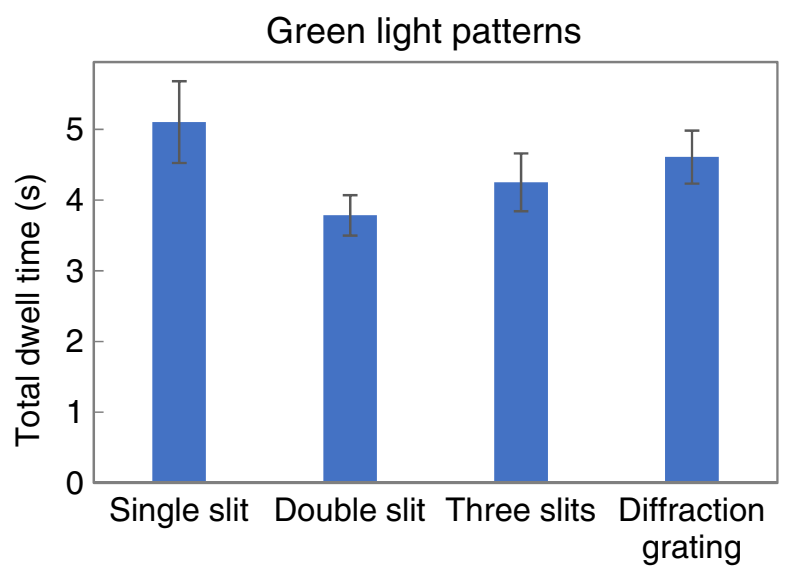

FIG. 9. Total dwell times for multiple-choice patterns (single slit, double slit, three slits, and diffraction grating) produced by the green light. Total dwell time is evaluated as the sum of dwell times obtained in questions Q5, Q6, and Q7.

pattern and double-slit pattern was statistically significant $(p<0.01)$.
[1] D. Halliday, R. Resnick, and J. Walker, Fundamentals of Physics Extended (John Wiley \& Sons, Hoboken, NJ, 2018).

[2] V. Mešić, K. Neumann, I. Aviani, E. Hasović, W. J. Boone, N. Erceg, V. Grubelnik, A. Sušac, D. S. Glamočić, M. Karuza, A. Vidak, A. Alihodžić, and R. Repnik, Measuring students' conceptual understanding of wave optics: A Rasch modeling approach, Phys. Rev. Phys. Educ. Res. 15, 010115 (2019).

[3] J. H. W. Hsiao and G. Cottrell, Two fixations suffice in face recognition, Psychol. Sci. 19, 998 (2008).

[4] A. Susac, R. J. Ilmoniemi, E. Pihko, J. Nurminen, and S. Supek, Early dissociation of face and object processing: A magnetoencephalographic study, Human Brain Mapp. 30, 917 (2009).

[5] A. Susac, M. Planinic, A. Bubic, L. Ivanjek, and M. Palmovic, Student recognition of interference and diffraction patterns: An eye-tracking study, Phys. Rev. Phys. Educ. Res. 16, 0020133 (2020).

[6] W. M. Roth, C. J. McRobbie, K. B. Lucas, and S. Boutonné, Why may students fail to learn from demonstrations? A social practice perspective on learning in physics, J. Res. Sci. Teach. 34, 509 (1997).

[7] E. Etkina, A. Van Heuvelen, D. T. Brookes, and D. Mills, Role of experiments in physics instruction-a process approach, Phys. Teach. 40, 351 (2002).

[8] B. S. Ambrose, P. S. Shaffer, R. N. Steinberg, and L. C. McDermott, An investigation of student understanding of single-slit diffraction and double-slit interference, Am. J. Phys. 67, 146 (1999).

[9] B. S. Ambrose, P. R. L. Heron, S. Vokos, and L. C. McDermott, Student understanding of light as an electromagnetic wave: Relating the formalism to physical phenomena, Am. J. Phys. 67, 891 (1999).

[10] B. S. Ambrose, Investigation of student understanding of the wave-like properties of light and matter, Ph.D. dissertation, Department of Physics, University of Washington, 1999, https://digital.lib.washington.edu/researchworks/handle/ $1773 / 9696$.

[11] K. Wosilait, P. R. L. Heron, P. S. Shaffer, and L. C. McDermott, Addressing student difficulties in applying a wave model to the interference and diffraction of light, Am. J. Phys. 67, S5 (1999).

[12] P. Colin and L. Viennot, Using two models in optics: Students' difficulties and suggestions for teaching, Am. J. Phys. 69, S36 (2001).

[13] L. Maurines, Geometrical reasoning in wave situations: The case of light diffraction and coherent illumination optical imaging, Int. J. Sci. Educ. 32, 1895 (2010).

[14] A. Coetzee and S. N. Imenda, Alternative conceptions held by first year physics students at a South African university of technology concerning interference and diffraction of waves, Res. High. Educ. J. 16, 13 (2012), http://www.aabri .com/manuscripts/121097.pdf.

[15] V. Mešić, A. Vidak, E. Hasović, and M. Dekić, University students' ideas about the role of the aperture and laser beam dimensions in formation of diffraction patterns, Eur. J. Phys. 40, 055701 (2019).

[16] R. Dai, J. C. Fritchman, Q. Liu, Y. Xiao, H. Yu, and L. Bao, Assessment of student understanding on light interference, Phys. Rev. Phys. Educ. Res. 15, 020134 (2019). 
[17] P. Hubber, Year 12 students' mental models of the nature of light, Res. Sci. Educ. 36, 419 (2006).

[18] V. Mešić, E. Hajder, K. Neumann, and N. Erceg, Comparing different approaches to visualizing light waves: An experimental study on teaching wave optics, Phys. Rev. Phys. Educ. Res. 12, 010135 (2016).

[19] S. K. Şengören, Turkish students' mental models of light to explain the single slit diffraction, and double slit interference of light: A cross-sectional study, J. Balt. Sci. Educ. 9, 61 (2010), http://oaji.net/articles/2014/987-1404741216 .pdf.

[20] S. K. Şengören, How do Turkish high school graduates use the wave theory of light to explain optics phenomena?, Phys. Educ. 45, 253 (2010).

[21] P. M. Sadler and R. H. Tai, Success in introductory college physics: The role of high school preparation, Sci. Educ. 85, 111 (2001).

[22] R. Leinonen, M. A. Asikainen, and P. E. Hirvonen, Peer discussions in lecture-based tutorials in introductory physics, Phys. Rev. Phys. Educ. Res. 13, 010114 (2017).

[23] N. S. Podolefsky, K. K. Perkins, and W. K. Adams, Factors promoting engaged exploration with computer simulations, Phys. Rev. ST Phys. Educ. Res. 6, 020117 (2010).

[24] E. Etkina and A. Van Heuvelen, Investigative science learning environment - A science process approach to learning physics, Research Based Reform of University Physics, edited by E. F. Redish and P. Cooney (2007), http://per-central.org/per_reviews/media/volume1/ISLE2007.pdf.

[25] R. W. Bybee, J. A. Taylor, A. Gardner, P. Van Scotter, J. Carlson Powell, A. Westbrook, and N. Landes, The BSCS 5E instructional model: Origins and effectiveness (2006), https://media.bscs.org/bscsmw/5es/bscs_5e_full_report .pdf.

[26] H. D. Young and R. A. Freedman, Sears and Zemansky's University Physics: With Modern Physics (Pearson Education, Upper Saddle River, NJ, 2016).

[27] R. Serway and J. Jewett, Physics for Scientists and Engineers with Modern Physics (Cengage Learning, Belmont, CA, 2018).

[28] R. D. Knight, Physics for Scientists and Engineers: A Strategic Approach with Modern Physics (Pearson Education, Upper Saddle River, NJ, 2017).

[29] D. C. Giancoli, Physics: Principles with Applications (Pearson Education, Upper Saddle River, NJ, 2014).

[30] R. J. Beichner, Testing student interpretation of kinematics graphs, Am. J. Phys. 62, 750 (1994).

[31] L. Ivanjek, A. Susac, M. Planinic, A. Andrasevic, and Z. Milin-Sipus, Student reasoning about graphs in different contexts, Phys. Rev. Phys. Educ. Res. 12, 010106 (2016).

[32] P. B. Kohl and N. D. Finkelstein, Student representational competence and self-assessment when solving physics problems, Phys. Rev. ST Phys. Educ. Res. 1, 010104 (2005).

[33] P. B. Kohl and N. D. Finkelstein, Effect of instructional environment on physics students' representational skills, Phys. Rev. ST Phys. Educ. Res. 2, 010102 (2006).

[34] A. D. Smith, J. P. Mestre, and B. H. Ross, Eye-gaze patterns as students study worked-out examples in mechanics, Phys. Rev. ST Phys. Educ. Res. 6, 020118 (2010).
[35] M. J. Tsai, H. T. Hou, M. L. Lai, W. Y. Liu, and F. Y. Yang, Visual attention for solving multiple-choice science problem: An eye-tracking analysis, Comput. Educ. 58, 375 (2012).

[36] A. Susac, A. Bubic, E. Kazotti, M. Planinic, and M. Palmovic, Student understanding of graph slope and area under a graph: A comparison of physics and nonphysics students, Phys. Rev. Phys. Educ. Res. 14, 020109 (2018).

[37] P. Klein, S. Küchemann, S. Brückner, O. ZlatkinTroitschanskaia, and J. Kuhn, Student understanding of graph slope and area under a curve: A replication study comparing first-year physics and economics students, Phys. Rev. Phys. Educ. Res. 15, 020116 (2019).

[38] P. Klein, A. Lichtenberger, S. Kuchemann, S. Becker, M. Kekule, J. Viiri, C. Baadte, A. Vaterlaus, and J. Kuhn, Visual attention while solving the test of understanding graphs in kinematics: An eye-tracking analysis, Eur. J. Phys. 41, 025701 (2019).

[39] P. Klein, J. Viiri, and J. Kuhn, Visual cues improve students' understanding of divergence and curl: Evidence from eye movements during reading and problem solving, Phys. Rev. Phys. Educ. Res. 15, 010126 (2019).

[40] A. Susac, A. Bubic, M. Planinic, M. Movre, and M. Palmovic, Role of diagrams in problem solving: An evaluation of eye-tracking parameters as a measure of visual attention, Phys. Rev. Phys. Educ. Res. 15, 013101 (2019).

[41] S. Küchemann, P. Klein, H. Fouckhardt, S. Gröber, and J. Kuhn, Students' understanding of non-inertial frames of reference, Phys. Rev. Phys. Educ. Res. 16, 010112 (2020).

[42] S. Brückner, O. Zlatkin-Troitschanskaia, S. Küchemann, P. Klein, and J. Kuhn, Changes in students' understanding of and visual attention on digitally represented graphs across two domains in higher education: A postreplication study, Front. Psychol. 11, 1 (2020).

[43] A. M. Madsen, A. M. Larson, L. C. Loschky, and N. S. Rebello, Differences in visual attention between those who correctly and incorrectly answer physics problems, Phys. Rev. ST Phys. Educ. Res. 8, 010122 (2012).

[44] A. Madsen, A. Rouinfar, A. M. Larson, L. C. Loschky, and N. S. Rebello, Can short duration visual cues influence students' reasoning and eye movements in physics problems?, Phys. Rev. ST Phys. Educ. Res. 9, 020104 (2013).

[45] A. Rouinfar, E. Agra, A. M. Larson, N. S. Rebello, and L. C. Loschky, Linking attentional processes and conceptual problem solving: Visual cues facilitate the automaticity of extracting relevant information from diagrams, Front. Psychol. 5, 1094 (2014).

[46] S. C. Chen, H. C. She, M. H. Chuang, J. Y. Wu, J. L. Tsai, and T. P. Jung, Eye movements predict students' computerbased assessment performance of physics concepts in different presentation modalities, Comput. Educ. 74, 61 (2014).

[47] A. Susac, A. Bubic, P. Martinjak, M. Planinic, and M. Palmovic, Graphical representations of data improve student understanding of measurement and uncertainty: An eye-tracking study, Phys. Rev. Phys. Educ. Res. 13, 020125 (2017).

[48] P. Klein, J. Viiri, S. Mozaffari, A. Dengel, and J. Kuhn, Instruction-based clinical eye-tracking study on the visual 
interpretation of divergence: How do students look at vector field plots?, Phys. Rev. Phys. Educ. Res. 14, 010116 (2018).

[49] J. Han, L. Chen, Z. Fu, J. Fritchman, and L. Bao, Eyetracking of visual attention in web-based assessment using the Force Concept Inventory, Eur. J. Phys. 38, 045702 (2017).

[50] M. Kekule and J. Viiri, Students' approaches to solving R-FCI tasks observed by eye-tracking method, Sci. Educ. 9, 117 (2018).
[51] https://www.itp.uni-hannover.de/fileadmin/arbeitsgruppen/ zawischa/static_html/multibeam.html.

[52] A. Susac, A. Bubic, J. Kaponja, M. Planinic, and M. Palmovic, Eye movements reveal students' strategies in simple equation solving, Int. J. Sci. Math. Educ. 12, 555 (2014).

[53] L. Ivanjek, P. Shaffer, M. Planinić, and L. McDermott, Probing student understanding of spectra through the use of a typical experiment used in teaching introductory modern physics, Phys. Rev. Phys. Educ. Res. 16, 010102 (2020). 